\title{
Europäische Rezeption der Berliner Hofoper und Hofkapelle von 1842 bis 1849
}

\author{
MATTHIEU CAILLIEZ, GRENOBLE
}

Gegenstand der Untersuchung ist die europäische Rezeption der Berliner Hofoper und Hofkapelle von 1842 bis 1849 anhand deutscher, französischer, italienischer, englischer, spanischer, belgischer und niederländischer Musikzeitungen. Im Jahr 1842 wird mit der Einführung regelmäßiger Sinfoniekonzerte eine bis heute fortwährende Tradition begründet. Im selben Jahr werden Giacomo Meyerbeer und Felix Mendelssohn Bartholdy als Generalmusikdirektor bzw. Dirigent der Sinfoniekonzerte verpflichtet. Der Tod des Dirigenten Otto Nicolai am 11. Mai 1849, knapp zwei Monate nach der Uraufführung seiner Oper Die lustigen Weiber von Windsor an der Berliner Hofoper, fällt mit dem Ende der analysierten Periode zusammen. Überdies stellen die Revolutionen von 1848 in der Geschichte Europas einen Wendepunkt dar. Die rege musikalische Aktivität dieser drei Dirigenten und Komponisten soll in diesem Beitrag besonders in den Blick genommen werden. Hierfür werden die vier wichtigsten deutschen, französischen, italienischen und englischen Musikzeitungen dieser Zeit, nämlich die Allgemeine musikalische Zeitung aus Leipzig, die Revue et Gazette musicale de Paris aus der französischen Hauptstadt, die Gazzetta musicale di Milano aus Mailand und The Musical World aus London als Hauptquelle dienen. Überdies finden folgende Musikzeitungen ebenfalls Einzug in die Untersuchung: die Neue Zeitschrift für Musik (Leipzig), die Berliner musikalische Zeitung, die Allgemeine Wiener Musik-Zeitung, Caecilia, eine Zeitschrift für die musikalische Welt (Mainz), La France musicale (Paris), Le Ménestrel (Paris), Il Pirata (Mailand), Bazar di Novità Artistiche, Letterarie e Teatrali (Mailand), L'Italia musicale (Mailand), Teatri, Arti e Letteratura (Bologna), La Iberia Musical (Madrid), La Belgique musicale (Brüssel), Caecilia, algemeen muzikaal tijdschrift van Nederland (Utrecht), usw. Damit werden teils europäische Bilder, teils stark differenzierte Nationalbilder über die Berliner Hofoper und Hofkapelle ersichtlich.

Im ersten Teil dieses Beitrags werden einige Tourneen von ausländischen Virtuosen und Sängern erwähnt, die vielfach nationale Interessen der verschiedenen Länder hervorheben. Des Weiteren soll die Berliner Hofoper und das Königstädtische Theater untersucht werden, die in dieser Zeit den sprachlichen und kulturellen Unterschied zwischen dem deutschen Theater und dem italienischen Theater verkörperten. Abschließend werden die zahlreichen Nachrichten über die drei deutschen Komponisten und Dirigenten Meyerbeer, Mendelssohn und Nicolai untersucht. 


\section{Tourneen ausländischer Virtuosen und Sänger}

Das Interesse der europäischen Musikzeitschriften für musikalische Nachrichten aus dem Berlin der 1840er Jahre war höchst unterschiedlich. Während La Iberia Musical aus Madrid und The Musical World aus London zum Beispiel meistens nur kurze und sporadische Mitteilungen machten - möglichweise aufgrund der größeren geographischen Entfernung -, informierte die deutschfreundliche Revue et Gazette musicale de Paris ihre Leser in wöchentlichem Rhythmus über die Konzerte und Opernaufführungen, die in der preußischen Hauptstadt gegeben wurden. Dieser Unterschied lässt sich wohl vor allem dadurch erklären, dass Maurice Schlesinger, Eigentümer der Revue et Gazette musicale de Paris, der Sohn Adolf Martin Schlesingers, des Gründers der Berliner Allgemeinen musikalischen Zeitung, war. Deshalb kennzeichnete die Pariser Zeitung ein besonderes Interesse für die musikalischen Ereignisse Deutschlands sowie die Zusammenarbeit mit berühmten deutschen Theoretikern, Musikkritikern und Komponisten wie Adolf Bernhard Marx, Ludwig Rellstab und Robert Schumann. Darüber hinaus bestand eine Kooperation der Revue et Gazette musicale de Paris mit Franz Liszt, der 1842 in Weimar zum Kapellmeister ernannt wurde. ${ }^{1}$ Umgekehrt ist dieses besondere Interesse für das Berliner Musikgeschehen in zwei weiteren Pariser Musikzeitschriften der Zeit - La France musicale, einer italienfreundlichen Zeitung und Le Ménestrel - nicht zu finden.

Die Berichterstattung europäischer Musikzeitungen über das Berliner Musikleben erfolgt oftmals aus einer national-geprägten Perspektive. So schilderte The Musical World am 30. Januar 1847 das Berliner Konzert des jungen Londoner Klavierspielers Ignatz Gibsone, der als hervorragender Künstler bezeichnet wird, wie folgt:

"A talented young pianist, Mr. Ignatz Gibsone, has arrived here from London. The communications
from London, Brussels, and the Rhenish towns on the concerts of Mr. Gibsone, drew the attention of
seeing the excellent artist appear amongst us. England shows now with such talents as Litolff, Balfe,
Wallace, Lord Westmoreland, Horsley, Bishop, Sloper, Macfarren, Parish Alvars, Bennett, Jewson,
Holmes, Hatton, Flowers, Cohan, \&c., that in music it is well qualified to rival with other nations."

Am 19. Juni 1847 werden ebenfalls die englischen Brüder Tivendell, ein Geiger und ein Klavierspieler, von The Musical World erwähnt. Der Geiger soll in Berlin ein Student von Mendelssohn und Spohr gewesen sein. Seine Kompositionen wurden von einer Berliner Zeitung gelobt - der entsprechende deutsche Bericht wird ins Englische übersetzt. ${ }^{3}$ Die Revue et Gazette musicale de Paris berichtet am 20. November 1842 im gleichen patriotischen Sinne vom Berliner Konzert einer jungen Sopranistin aus Lothringen:

„Berlin, 6 novembre. - On annonce le prochain début à notre Théâtre-Italien de la première et seule élève que la célèbre cantatrice madame Dell'Occa Schoberlechner ait jamais formée, mademoiselle

1 Siehe die Liste der Herausgeber der Revue et Gazette musicale de Paris im Jahr 1844.

2 The Musical World 22, H. 5 (30. Januar 1847), S. 70.

3 The Musical World 22, H. 25 (19. Juni 1847), S. 401. 
Pauline Delaroche, native de Nancy, en France, et âgée de dix-huit ans. Cette jeune personne, qui possède, dit-on, une voix de soprano aussi remarquable par sa suave sonorité que par son étendue, a chanté avant-hier, dans un concert donné à la cour, un air de Meyerbeer et un duo de Mercadante avec madame Dell'Occa Schoberlechner, qui lui ont valu les suffrages unanimes de l'illustre assemblée."4

Die Berliner Tourneen der Sängerinnen der Garcia-Familie, in erster Linie Pauline Viardots, wurden oft in der europäischen Presse kommentiert. ${ }^{5}$ So profitierte auch die Cousine Viardots von deren Anwesenheit:

„BERLIN. - (From a Correspondent.) - Mademoiselle Antonina di Mendi, cousin of Madame Malibran and Mademoiselle Viardot Garcia, who made so favourable an impression upon our aristocratic dilettanti during the latter part of last season, has been paying a visite artistique to Berlin, where she has been received with the most flattering marks of approbation [...]. She has been singing with the greatest success at court, in company with Mademoiselle Viardot Garcia; the critics give their general assent, that age alone is required to make her a worthy vocal member of the Garcia family. Other foreign journals also announce a similar success having befallen this charming young artist in a recent tour through Belgium. ${ }^{\text {"6 }}$

In der La Iberia Musical aus Madrid wurden am 27. August 1843 die großen Talente Pauline Viardots, die gerade in Berlin unter der Leitung Meyerbeers gesungen hatte, gelobt. Der nationale Stolz über die berühmte Tochter des spanischen Tenors Manuel Garcia ist dabei nicht zu übersehen. ${ }^{7}$

Neben den Aufenthalten "ihrer Musiker" in Berlin berichteten die Musikzeitungen auch über ausländische Künstler, die zuvor in dem jeweiligen Land zu Gast waren und der heimischen Presse deshalb bekannt waren sowie über Künstler, die bald in ihrem eigenen Land auftreten würden. Dies trifft beispielsweise auf die Zeitung Caecilia, algemeen muzikaal tijdschrift van Nederland aus Utrecht zu, die in ihrer Ausgabe vom 15. Juni 1848 das einzige Konzert der Sängerin Garcia-Torres und des belgischen Cellisten François Demunck in Berlin erwähnte und in diesem Zusammenhang auf die bevorstehende Tournee in den Niederlanden hinwies. ${ }^{8}$ Ebenso merkte die französische Revue et Gazette musicale de Paris am 9 . Januar 1842 an, dass die italienische Sängerin Laura Assandri, die gerade vom Berliner Publikum gefeiert wurde, am Pariser Théâtre-Italien Karriere gemacht hatte. So wird indirekt das Niveau des eigenen musikalischen Lebens unterstrichen: „Mademoiselle Laure Assandri, que nous avons vue naguère au Théâtre-Italien de Paris, et qui depuis a fait de grands progrès, est la cantatrice favorite des Berlinois; on la compare à mademoiselle Sontag, et on la rappelle à chaque représentation." ${ }^{\prime 9}$

4 Revue et Gazette musicale de Paris 9, H. 47 (20. November 1842), S. 460.

5 Il Pirata 13, H. 90 (26. Januar 1848), S. 366: „Berlino, 1 gennajo. La ricomparsa della Juive d'Halevy ave[v]a attirato molto concorso al teatro Reale, quantunque i prezzi fossero aumentati. La Viardot-Garcia sostenne la parte di Rachele."

6 The Musical World 22, H. 4 (23. Januar 1847), S. 56.

7 La Iberia Musical 2, H. 33 (27. August 1843), S. 280.

8 Caecilia, algemeen muzikaal tijdschrift van Nederland 5, H. 12 (15. Juni 1848), S. 106.

9 Revue et Gazette musicale de Paris 9, H. 2 (9. Januar 1842), S. 15. 
Außerdem thematisierte Florentius Cornelis Kist das musikalische Leben Berlins in langen Artikeln, die in Caecilia, algemeen muzikaal tijdschrift van Nederland veröffentlicht wurden. Der Chefredakteur bewies damit ein verstärktes Interesse für die verschiedensten musikalischen Bereiche des Nachbarlandes. ${ }^{10}$ Auch die Leipziger Allgemeine musikalische Zeitung erweist sich - mit der wiederholten Veröffentlichung sehr langer und ausführlicher Berichte - als eine sehr zuverlässige und umfangreiche Informationsquelle über das Berliner Musikgeschehen. ${ }^{11}$

\section{Berliner Hofoper und Königstädtisches Theater: deutsches Theater versus italienisches Theater}

In der ersten Hälfte des 19. Jahrhunderts war die Verbreitung der italienischen Oper in Berlin besonders präsent. Insgesamt wurden vor 1850 nicht weniger als 65 italienische Opern, die zwischen 1810 und 1850 andernorts uraufgeführt wurden, in Berlin aufgeführt. Dazu zählen 21 Opern Donizettis, 19 Opern Rossinis, sieben Opern Bellinis, sechs Opern Mercadantes, jeweils drei Opern Meyerbeers und Verdis, zwei Opern Luigi Riccis und Otto Nicolais sowie eine Oper Pacinis und Coppolas. ${ }^{12}$

16 der 19 vor 1850 in Berlin aufgeführten Opern Rossinis wurden im Königstädtischen Theater gegeben, was die führende Rolle dieses Theaters in der Berliner Verbreitung der italienischen Oper bestätigt. Mehr noch als für die Opern Rossinis hat das Königstädtische Theater entscheidend zur Verbreitung der Opern Donizettis in Berlin beigetragen. So wurden alle 21 Opern Donizettis, die in Berlin vor 1850 aufgeführt wurden, in diesem Theater gegeben. Nur acht davon wurden auch in der Hofoper aufgeführt. Während die ersten Aufführungen der Opern Donizettis im Königstädtischen Theater in den 1830er Jahren hauptsächlich auf Deutsch stattfanden, wurden die Aufführungen seiner Opern in den 1840er Jahren auf Italienisch gegeben. Parallel wurden regelmäßig italienische Saisons organisiert. 17 Opern des Bergameser Komponisten kamen so zwischen 1841 und 1847 auf dieser Bühne auf Italienisch zur Aufführung. Hierbei handelt es sich um die Periode, die uns im Folgenden besonders beschäftigen wird. Die wichtige Verbreitung der Opern Donizettis im Berlin der 1840er Jahre entspricht dem damaligen Erfolg der Opern des Komponisten im deutschsprachigen Raum. Ein Erfolg, den Franz Liszt in der Ausgabe der Neuen Zeitschrift für Musik des 4. August 1854 mit Verbitterung kommentierte. Ebenso wie die Opern Donizettis wurden die sieben Opern Bellinis, die in Berlin gegeben

10 Florentius Cornelis Kist, „Verhandelingen. Reize door Duitschland in het Jaar 1843. [...] Berlijn”, in: Caecilia, algemeen muzikaal tijdschrift van Nederland 5, H. 19 (1. Oktober 1848), S. 155-156.

11 Siehe zum Beispiel: Allgemeine musikalische Zeitung 44, H. 1 (5. Januar 1842), S. 12-16; Allgemeine musikalische Zeitung 44, H. 3 (19. Januar 1842), S. 63-68; Allgemeine musikalische Zeitung 44, H. 7 (19. Februar 1842), S. 143-146; Allgemeine musikalische Zeitung 44, H. 8 (23. Februar 1842), S. 162-166; usw.

12 Siehe: Matthieu Cailliez, La Diffusion du comique en Europe à travers les productions d'opere buffe, d'opérascomiques et de komische Opern (France - Allemagne - Italie, 1800-1850), Dissertation, Universität Bonn 2016, <http://hss.ulb.uni-bonn.de/2016/4527/4527.htm> (01.04.2020), S. 197-202, S. 647-651. 
wurden, alle im Königstädtischen Theater aufgeführt. In der Hofoper wurden hingegen nur drei Opern des Komponisten auf Deutsch gegeben.

An der Berliner Hofoper wurden vor 1850 insgesamt 24 zeitgenössische italienische Opern von sieben Komponisten auf Deutsch aufgeführt. Im Vergleich dazu wurden im Königstädtischen Theater gleichzeitig 57 Opern von zehn Komponisten auf die Bühne gebracht, davon 28 Opern auf Deutsch und 45 Opern auf Italienisch. Damit ist die Zahl italienischer Opern, die am Königstädtischen Theater zwischen 1824 und 1850 gegeben wurden, ungefähr zweieinhalb Mal höher als die Anzahl der an der Hofoper zwischen 1818 und 1850 aufgeführten Opern.

Wenig überraschend berichteten die italienischen Musikzeitungen sehr regelmäßig über die italienischen Aufführungen im Königstädtischen Theater. ${ }^{13}$ So wird man in der Gazzetta musicale di Milano vom 16. Februar 1848 darüber unterrichtet, dass die letzte Aufführung von Bellinis Oper I Puritani eine der besten Aufführungen der Saison in der sogenannten Italienischen Oper von Berlin war. Die Mailänder Musikzeitung stellte ihrer Leserschaft eine italienische Übersetzung eines Berichts einer Berliner Musikzeitung zur Verfügung, in dem die verschiedenen Sänger und Sängerinnen der am 22. Januar gegebenen Aufführung bewertet werden. Bemerkenswert ist, dass die italienischen Saisons in Berlin lange im Voraus angekündigt wurden: so informierten die italienischen Musikzeitungen ihre Leser über das Rekrutieren der Mitglieder der Sängertruppe. ${ }^{14}$ In der Zeitung Teatri, Arti e Letteratura aus Bologna war am 14. März 1844 Folgendes zu lesen:

„L'Agenzia Teatrale di Cazzioletti e Benelli di Milano scritturò pel Reale Teatro Italiano di Berlino, dal $1 .{ }^{\circ}$ settembre prossimo a tutto maggio 1845 , le prime donne soprano signore Luigia Schieroni Nulli e Maddalena Belloni, la prima donna contralto signora Elisa Bendini, le comprimarie signore Carolina Remorini e Giovanna Ricca, i primi tenori signori Fortunato Borioni e Giovanni Landi, i primi bassi signori Cleto Capitini e Giovanni Mitrovich, per primo buffo il signor Simone Grandi, per altro primo basso Temistocle Santi-Silva, e per altro primo tenore Cavirani Alessandro, e dal primo del corrente mese a tutto maggio del corrente anno il primo tenore signor Luigi Donati. ${ }^{\prime 15}$

In den italienischen Zeitungen und Musikzeitschriften der 1840er Jahre gab es tausende Meldungen dieser Art. Sie ermöglichten den zahlreichen Akteuren der italienischen Musikindustrie eine präzise Übersicht der weltweit organisierten italienischen Saisons. Hunderte davon betreffen allein die italienischen Saisons in Berlin. Die folgende Nachricht, die am 18. Januar 1848 in der Mailänder Zeitung /l Pirata gedruckt wurde, erwähnt mehr als ein Jahr im Voraus ein Teil der Mitglieder der in Berlin engagierten italienischen Truppe und nennt sogar den Namen des verpflichteten Dirigenten: „L'Agente Teatrale Bonola scritturò per Berlino, dalla metà di agosto prossimo a tutto il mese di aprile 1849, il Maestro-Direttore signor Giuseppe De Barbieri, e i

13 Bazar di Novità Artistiche, Letterarie e Teatrali 7 (1847), S. 7, 20, 32, 36, 80, 96, 100, 102-103, 108, 111, 124, 132, $144,148,164,171$, usw.

14 Gazzetta musicale di Milano 7, H. 7 (16. Februar 1848), S. 54.

15 Teatri, Arti e Letteratura 41, H. 1049 (14. März 1844), S. 14. 
primi tenori signori Domenico Labaccetta (riconfermato per la terza volta) e il sig. Gaetano Pardini (parimenti riconfermato)." ${ }^{\prime 16}$

Einige Nachrichten sind nichts anderes als unterhaltende Anekdoten, wie der folgende Auszug der Musikzeitung aus Bologna exemplarisch beweist:

„All'ultima rappresentazione dell'opera che davasi in questa città, allorchè andavasi a compire il terzo atto dei Capuleti e Montecchi, Romeo, vicino al feretro della sua cara Giulietta, cantata la sua aria, attendeva che la sua diletta si rialzasse per cantare essa pure la sua grand'aria di morte. Niuna risposta. Giulietta è immobile. Romeo ripete la sua frase e s'inchina dolcemente all'orecchio di Giulietta dicendole: "Alzatevi, signora Kaiser". E di nuovo nessuna risposta, allora Romeo fa cenno ad un uomo della scena, si chiama il direttore, si teme qualche disgrazia, ed il pubblico è impaziente. II direttore arriva; un russare scusibilissimo uscito dal fondo del feretro annunziò al pubblico che la signora Kaiser era addormentata di profondo sonno. II pubblico partì ridendo, la tela calò e tutto finì nelle risa.. ${ }^{17}$

Im Königstädtischen Theater wurden sogar französische Opéras-comiques und Grands Opéras, wie Zampa von Ferdinand Herold oder Robert le diable des deutschen Komponisten Meyerbeer, auf Italienisch aufgeführt. ${ }^{18}$ Die italienische Truppe sang manchmal im deutschen Theater, das heißt in der Berliner Hofoper, wie z. B. im Januar 1848 im Falle einer Aufführung von Rossinis Barbiere di Siviglia. ${ }^{19}$

Die deutschen Opern, die auf die Bühne der Hofoper gebracht wurden, erweckten nur wenig Interesse in den französischen und italienischen Musikzeitungen dieser Zeit. Umgekehrt findet man zahlreiche Berichte über Meyerbeers französische Opern, die dort auf Deutsch aufgeführt wurden. Die Opern und Ballette französischer Komponisten, wie Daniel-François-Esprit Auber, Fromental Halévy oder Adolphe Adam, blieben auch nicht unerwähnt.20 Der folgende Auszug aus der Gazzetta musicale di Milano zeugt vom leidenschaftlichen Interesse des Berliner Publikums für die Meisterwerke der Vergangenheit, während das italienische Publikum nur für zeitgenössische Opern schwärmte:21

„BERLINO. - L'intendenza de' teatri regi di quella capitale, uniformandosi al desiderio manifestato dal ministro dell'interno, di vedere eseguiti di tempo in tempo sul teatro della Grande-Opera dei capolavori antichi, ha fatto porre nel repertorio di quel teatro le sei Opere seguenti: Arminio di Hasse; Arianna di Haendel; Proserpina ed Alceste di Lulli; Cajo Mario di Jommelli e la Principessa fedele di Scarlatti. - Nella sola Italia, i capolavori antichi saranno sempre obbliati? e noi non applaudiremo che alla musica di moda?" 22

16 Il Pirata 13, H. 85 (15. Januar 1848), S. 346.

17 Teatri, Arti e Letteratura 42, H. 1074 (5. September 1844), S. 7.

18 Caecilia, algemeen muzikaal tijdschrift van Nederland 5, H. 6 (15. März 1848), S. 56: „In het Italiaansche tooneel te Berlijn is Robert le Diable met schitterend effect uitgevoerd en den 24. Febr. zijn 2 Aktes der Hugenoten en de 3e van Othello, van Rossini, ten benefite van Mad. Viardot Garcia, die daar steeds veel opgang maakt, gegeven."

19 Gazzetta musicale di Milano 7, H. 5 (2. Februar 1848), S. 38.

20 Revue et Gazette musicale de Paris 9, H. 17 (24. April 1842), S. 183.

21 Bernd Zegowitz, Der Dichter und der Komponist. Studien zu Voraussetzungen und Realisationsformen der Librettoproduktion im deutschen Opernbetrieb der ersten Hälfte des 19. Jahrhunderts, Würzburg 2012, S. 38: "Dass in der Theaterorganisation keine kontinuierliche Nachfrage nach neuen Opern vorhanden war, lag einerseits an der speziellen Struktur der deutschen Theaterlandschaft mit ihrer Aufteilung in Hof- und Stadttheater, andererseits auch am Spielsystem der deutschen Opernhäuser - Repertoire statt ,stagione'."

22 Gazzetta musicale di Milano 1, H. 12 (20. März 1842), S. 50. 


\section{Meyerbeer, Mendelssohn und Nicolai}

Mehr als jeder andere deutsche Komponist stand Giacomo Meyerbeer unter der ständigen Beobachtung der europäischen Musikpresse. Jeder seiner Schritte zwischen Paris und Berlin wurde kommentiert. Abwechselnd erwartete man inn in der französischen oder preußischen Hauptstadt.23 Beispielsweise berichtete La France musicale am 20. August 1843: "Meyerbeer est parti le 10 août seulement de Berlin, pour aller aux eaux de Schwalbach, dans le duché de Nassau, où se trouve sa famille. Le célèbre artiste ne sera de retour à Paris que vers le milieu de septembre." ${ }^{24}$ Sein Zeitplan war allen bekannt und es wurde gern über seine zukünftigen Opern, welche die internationalen Erfolge von Robert le diable und Les Huguenots wiederholen sollten, spekuliert. ${ }^{25}$ Laut der Revue et Gazette musicale de Paris soll der Erfolg dieser Oper in Berlin im Jahr 1842 sehr beeindruckend gewesen sein:

„Hier enfin a eu lieu, au théâtre royal du Grand-Opéra de notre capitale, la première représentation des Huguenots, de Meyerbeer. Le rôle de Valentine était rempli par la célèbre Mme Schroeder-Devrient, que la direction du théâtre, comme on sait, avait appelée de Dresde exprès pour lui confier ce personnage, qui exige à la fois une grande cantatrice et une comédienne consommée. L'illustre auteur tenait lui-même le piano. Cet opéra a obtenu un succès colossal, et à la fin du spectacle la salle a retenti longtemps des cris de vive Meyerbeer ! Madame Schroeder-Devrient a été rappelée trois fois sur la scène." 26

Die Nähe des Komponisten zum preußischen König wurde in der Presse oft betont. Manchmal handelt es sich um Werke, die ausdrücklich vom König bestellt wurden. ${ }^{27}$ In anderen Fällen wurden seine institutionellen Funktionen hervorgehoben.

Die Berliner Konzerte Mendelssohns als Pianist und Dirigent sowie die Uraufführungen seiner letzten Werke wurden oft thematisiert. ${ }^{28}$ Das Projekt eines Berliner Konservatoriums unter seiner Leitung wurde zwischen 1842 und 1844 mehrmals in französischen und in italienischen Medien besprochen. ${ }^{29}$ So liest man am 7. März 1844 in Teatri, Arti e Letteratura: „Sua Maestà il re di

23 Revue et Gazette musicale de Paris 9, H. 40 (2. Oktober 1842), S. 398: „Berlin. [...] - M. Meyerbeer, directeurgénéral de la musique du roi, est attendu pour le 1er décembre." Gazzetta musicale di Milano 3, H. 7 (18. Februar 1844), S. 30; La France musicale 9, H. 3 (18. Januar 1846), S. 22: „Meyerbeer a quitté Paris cette semaine ; il est dans ce moment sur la route de Berlin, où il va rejoindre sa famille." Le Ménestrel 14, H. 34 (25. Juli 1847), S. 3-4; Le Ménestrel 14, H. 49 (7. November 1847), S. 3; usw.

24 La France musicale 6, H. 34 (20. August 1843), S. 275.

25 La Iberia Musical 2, H. 1 (1. Januar 1843), S. 8: „El célebre compositor Meyerbeer partirá en los últimos días del mes de diciembre para Berlín: Meyerbeer está sumamente disgustado por lo mucho que se retarda la presentación en la escena de la nueva ópera El Profeta, la cual después de variar muchos parajes de ella, se encuentra sin artistas que la ejecuten."

26 Revue et Gazette musicale de Paris, H. 22 (29. Mai 1842), S. 231.

27 La France musicale 7, H. 2 (14. Januar 1844), S. 14: „M. Meyerbeer a été chargé, par le roi de Prusse, de composer un opéra pour l'ouverture du théâtre royal de Berlin. Cette inauguration aura lieu le 15 octobre prochain. Heureux roi de Prusse!"

28 The Musical World 17 (1842), S. 37, 69-70, 166; Revue et Gazette musicale de Paris 9 (1842), S. 23, 47, 197, 216, 223, 407, 510, 519; Gazzetta musicale di Milano 1, H. 7 (1842), S. 28, 106; La Iberia Musical 2, H. 22 (28. Mai 1843), S. 176; usw.

29 Revue et Gazette musicale de Paris 9, H. 26 (26. Juni 1842), S. 270: „Berlin, 10 juin. — Les statuts du Conservatoire 
Prussia ha assegnati 300.000 talleri per la fondazione di un Conservatorio di Musica a Berlino, che verrà organizzato sulle basi stesse di quello di Parigi. Il signor Mendelssohn verrà chiamato alla direzione." 30 Der Tod des Komponisten 1847 wurde in ganz Europa thematisiert, u. a. in London, Paris und Mailand. ${ }^{31}$ Sein Geburtstag und sein Todestag boten den Anlass für zahlreiche Konzerte und Veranstaltungen in Berlin, wie die Gazzetta musicale di Milano mehrmals

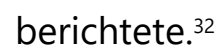

Im Gegensatz dazu wurde die Berliner Tätigkeit Otto Nicolais nicht im vergleichbaren Umfang von der europäischen Presse behandelt. Außerhalb der deutschsprachigen Welt interessierte man sich für Nicolai hauptsächlich in Italien ${ }^{33}$, da der Komponist um 1840 vier italienischen Opern geschrieben hatte, die ihm dort eine gewisse Bekanntheit verschafften. Die Uraufführung seiner komischen Oper Die lustigen Weiber von Windsor am 9. März 1849 im Königlichen Opernhaus fand nur wenig Beachtung im Ausland. ${ }^{34}$ Sein Tod in Berlin am 11. Mai 1849 wurde in den meisten deutschsprachigen Musikzeitungen gemeldet, blieb aber, von wenigen Aus-

royal de musique de Berlin, dont la création a été ordonnée dans le mois de novembre dernier, viennent d'être arrêtés par le conseil du ministère de l'instruction publique. En voici la substance: $1^{\circ}$ Le Conservatoire occupera un bâtiment dépendant du palais de l'Université royale de Berlin ; $2^{\mathrm{e}}$ il y aura cent élèves (cinquante de chaque sexe) qui, tous, recevront l'enseignement gratis, et dont quarante (vingt garçons et vingt jeunes filles) seront logés, nourris et vêtus aux frais de l'Etat; $3^{\circ}$ le nombre des professeurs est fixé à dix-huit, et sera successivement porté à vingt-quatre ; $4^{\circ}$ tous les doubles des œuvres musicales et des ouvrages théorétiques [sic] sur la musique, qui se trouvent dans les bibliothèques royales ou publiques du royaume, seront remis au Conservatoire pour former le noyau de sa future bibliothèque ; $5^{\circ}$ une somme de 100,000 thalers (environ $300,000 \mathrm{fr}$.), à prendre sur le budget du ministère de l'instruction publique, est affectée à l'achat du matériel et aux frais d'installation. M. le comte de Redern, intendant-général de la musique en Prusse, et M. Félix Mendelssohn-Bartholdy, maître de chapelle du roi, sont nommés, le premier, chef, et l'autre, directeur du Conservatoire royal de musique de Berlin. Le Conservatoire sera inauguré et commencera ses travaux dans le mois de janvier ou de février de l'année prochaine."

30 Teatri, Arti e Letteratura 41, H. 1048 (7. März 1844), S. 6.

31 Wiener allgemeine Musik-Zeitung 7, H. 134 (9. November 1847), S. 540; Allgemeine musikalische Zeitung 49, H. 45 (10. November 1847), S. 769-770, 776-777; The Musical World 22, H. 46 (13. November 1847), S. 718-720; Revue et Gazette musicale de Paris 14, H. 46 (14. November 1847), S. 374; Neue Zeitschrift für Musik 27, H. 40 (15. November 1847), S. 239-240; L'Italia musicale 1, H. 20 (17. November 1847), S. 157; Le Ménestrel 14, H. 51 (21. November 1847), S. 3. Siehe: Caecilia, algemeen muzikaal tijdschrift van Nederland 5 (1848), S. 3f., 15, 21f., 31f., 49f., 66-68, 86f., 99f., 108f., 118-120, 128, 130, 133-136, 191ff., 199f.; Caecilia, eine Zeitschrift für die musikalische Welt, 27 (1848), S. 115f., 129-144.

32 Gazzetta musicale di Milano 7, H. 2 (12. Januar 1848), S. 15; Gazzetta musicale di Milano 7, H. 8 (23. Februar 1848), S. 62. Siehe: Revue et Gazette musicale de Paris 15, H. 8 (13. Februar 1848), S. 55; Revue et Gazette musicale de Paris 15, H. 48 (26. November 1848), S. 370.

33 Teatri, Arti e Letteratura 41, H. 1073 (29. August 1844), S. 215f.: „Il maestro Niccolaj [sic] è partito da Berlino per Konisberga onde dirigere la sua grande ouverture sacra, sopra un corale in occasione della festa centennaria di quell'Università."; Teatri, Arti e Letteratura 42, H. 1079 (10. Oktober 1844), S. 48f.; Revue et Gazette musicale de Paris 15, H. 52 (24. Dezember 1848), S. 401.

34 Caecilia, algemeen muzikaal tijdschrift van Nederland 6, H. 7 (1. April 1849), S. 72; Revue et Gazette musicale de Paris 16, H. 12 (25. März 1849), S. 96: „On a donné les Commères de Windsor, opéra-comique de M. le maître de chapelle Nicolaï. Le libretto, d'après Shakspeare [sic], est écrit avec une certaine verve de gaieté et de joyeuse humeur que l'on ne retrouve pas toujours dans les inspirations du compositeur. Du reste, il y a de la facilité; les parties de chant sont habilement traitées; quelques morceaux d'un mérite supérieur ont assuré le succès de l'ouvrage." 
nahmen abgesehen, nahezu unbemerkt im übrigen Europa. ${ }^{35}$ So ist man in Bologna sieben Monate später immer noch nicht darüber benachrichtigt worden: "Il maestro Nicolay trovasi ora a Berlino, onde porre in iscena una sua opera nuova intitolata Le Banni, per il giorno anniversario di S. M. la Regina. ${ }^{\prime 36}$

Zusammenfassend lässt sich sagen, dass die europäische Musikpresse der 1840er Jahre eine sehr umfangreiche Fülle von Informationen über das musikalische Leben in Berlin zu dieser Zeit bietet. Auch wenn jedes Land seine eigenen Musiker und das jeweilige musikalische Repertoire nicht unparteiisch bevorzugt, kann eine gründliche und wesentliche Untersuchung dieses breiten Korpus einen wichtigen Beitrag zur Erforschung der Berliner Musikgeschichte im europäischen Kontext leisten.

Zitation: Matthieu Cailliez, „Europäische Rezeption der Berliner Hofoper und Hofkapelle von 1842 bis 1849”, in: Freie Beiträge zur Jahrestagung der Gesellschaft für Musikforschung 2019, hrsg. von Nina Jaeschke und Rebecca Grotjahn (= Musikwissenschaft: Aktuelle Perspektiven. Bericht über die Jahrestagung der Gesellschaft für Musikforschung 2019 in Paderborn und Detmold, Bd. 1), Detmold 2020, S. 55-64, DOI: 10.25366/2020.50.

35 Signale für die musikalische Welt 7, H. 25 (15. Mai 1849), S. 200; Neue Berliner Musikzeitung 3, H. 20 (16. Mai 1849), S. 160; Revue et Gazette musicale de Paris 16, H. 20 (20. Mai 1849), S. 159; Neue Zeitschrift für Musik 30, H. 44 (31. Mai 1849), S. 240; Caecilia, algemeen muzikaal tijdschrift van Nederland 6, H. 13 (1. Juli 1849), S. 123f.

36 Teatri, Arti e Letteratura 52, H. 1304 (6. Dezember 1849), S. 39. Siehe: La Belgique musicale 10, H. 49 (6. Dezember 1849), S. 4. 


\section{Abstract}

The subject of this contribution is the European reception of the Berlin Royal Opera House and Orchestra from 1842 to 1849 based on German, French, Italian, English, Spanish, Belgian and Dutch music journals. The institution of regular symphony concerts, a tradition continuing to the present, was initiated in 1842. Giacomo Meyerbeer and Felix Mendelssohn Bartholdy were hired as general music directors respectively conductors for the symphony concerts in the same year. The death of the conductor Otto Nicolai on 11th May 1849, two months after the premiere of his opera Die lustigen Weiber von Windsor, coincides with the end of the analysed period, especially since the revolutions of 1848 in Europe represent a turning point in the history of the continent. The lively music activities of these three conductors and composers are carefully studied, as well as the guest performances of foreign virtuosos and singers, and the differences between the Berliner Hofoper and the Königstädtisches Theater.

\section{Kurzvita}

Matthieu Cailliez ist wissenschaftlicher Mitarbeiter an der Universität Grenoble Alpes. 2014 promovierte er im Rahmen des Trinationalen Graduierten Kollegs der Universitäten Bonn, Paris-Sorbonne und Florenz "Gründungsmythen Europas in Literatur, Kunst und Musik". Der Titel seiner Doktorarbeit lautet La Diffusion du comique en Europe à travers les productions d'opere buffe, d'opéras-comiques et de komische Opern (France - Allemagne - Italie, 1800-1850). 


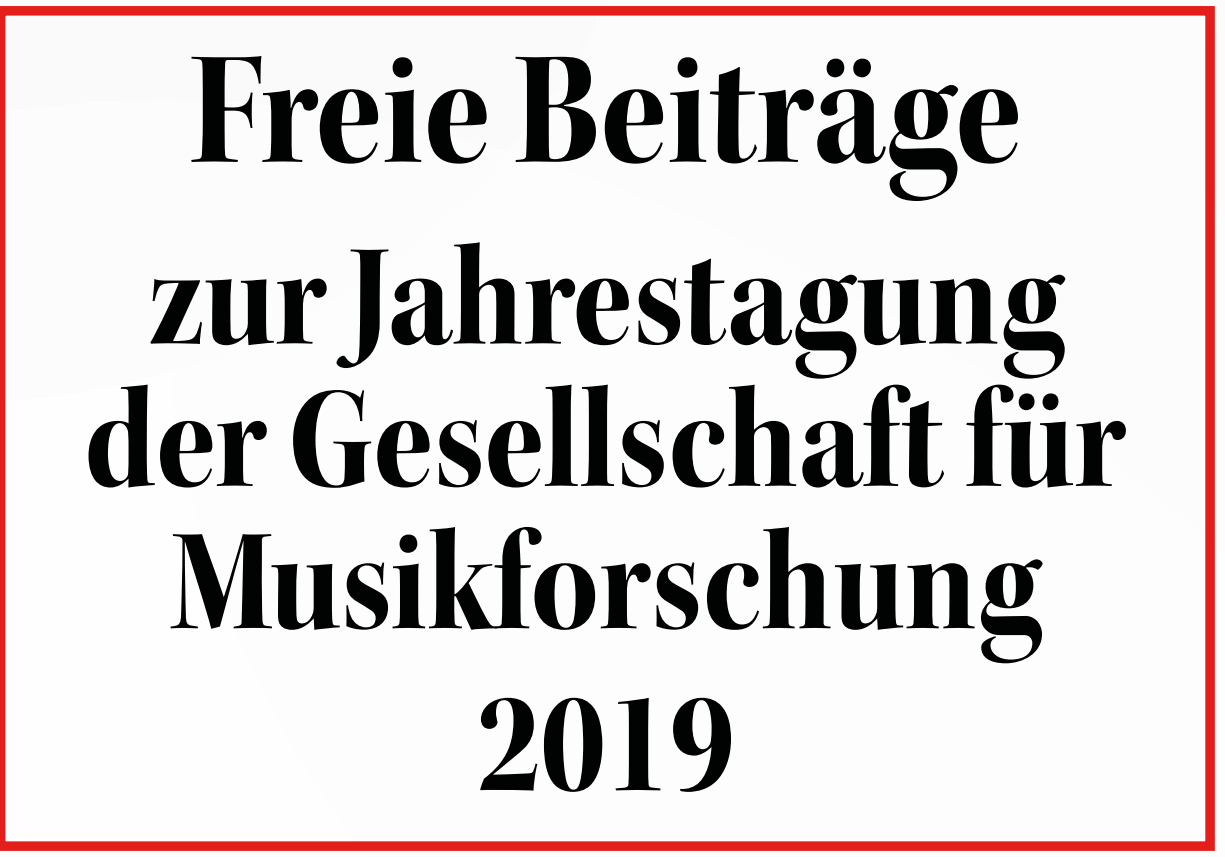

Herausgegeben von Nina Jaeschke und Rebecca Grotjahn

Musikwissenschaft: Aktuelle Perspektiven 1 
Freie Beiträge 


\section{Musikwissenschaft: Aktuelle Perspektiven}

Bericht über die Jahrestagung der Gesellschaft für Musikforschung 2019 in Paderborn und Detmold

Herausgegeben von Rebecca Grotjahn und Nina Jaeschke

Band 1 


\section{Freie Beiträge}

\section{zur Jahrestagung der Gesellschaft für Musikforschung 2019}

Herausgegeben von Nina Jaeschke und Rebecca Grotjahn

Detmold: Musikwissenschaftliches Seminar der Universität Paderborn und der Hochschule für Musik Detmold 2020 
DOI: $10.25366 / 2020.42$

Online-Version verfügbar unter der Lizenz: Urheberrecht 1.0, $<$ https://rightsstatements.org/page/InC/1.0/?language=de>

Bibliografische Information der Deutschen Nationalbibliothek

Die Deutsche Nationalbibliothek verzeichnet diese Publikation in der Deutschen Nationalbibliografie; detaillierte bibliografische Daten sind im Internet über http://dnb.d-nb.de abrufbar.

\section{Impressum}

Redaktion: Nina Jaeschke, Rebecca Grotjahn und Jonas Spieker Satz: Nina Jaeschke

(C) Musikwissenschaftliches Seminar der Universität Paderborn und der Hochschule für Musik Detmold 2020 


\section{INHALT}

Vorwort $\quad$ IX

Komponieren für das Radio: Akteure, Diskurse, Praktiken $\quad 1$

Musikwissenschaft - Feminismus - Kritik: Ein Generationenaustausch 6

\section{Stefan Alschner}

Der Wagner-Sänger Joseph Aloys Tichatschek - Vom Nachlass zum Netzwerk

\section{Alenka Barber-Kersovan}

Songs for the Goddess. Das popmusikalische Neo-Matriarchat zwischen Ethno-Beat,

erfundenen Traditionen und kommerzieller Vermarktung

Elias Berner, Julia Jaklin, Peter Provaznik, Matej Santi, Cornelia Szabó-Knotik

Musikgeschichte anders erzählen? Das Beispiel der 1970er in Österreich.

Musikhistoriographie in der Zeit der Digitalisierung

\section{Mauro Fosco Bertola}

„Ein Laut so klagevoll”. Lohengrin zwischen Richard Wagner und Salvatore Sciarrino

\section{Matthieu Cailliez}

Europäische Rezeption der Berliner Hofoper und Hofkapelle von 1842 bis 1849

\section{lacopo Cividini}

Zwischen klassischer Musikphilologie und angewandter Informatik:

Die Digitale Mozart-Edition (DME) der Stiftung Mozarteum Salzburg

\section{Marko Deisinger}

Fortschrittliche Technologie im Dienste eines Antimodernisten.

Heinrich Schenker und der österreichische Rundfunk

\section{Norbert Dubowy}

Vom Kritischen Bericht zur Kritischen Dokumentation am Beispiel der Digital-interaktiven Mozart-Edition

\section{Markus Engelhardt}

Musik zwischen Nation Building und Internationalität. Italien um 1900

\section{Maryam Haiawi}

Das Oratorium im Spannungsfeld der Konfessionen: 


\section{Judith I. Haug}

"Manch eine*r liegt, morgens noch trunken, im Rosengarten" - Rekonstruktionen

osmanischer Musikgeschichte in Gesangstextsammlungen

\section{Renate Koch}

Marcel Prawy und das erste Broadway-Musical im Österreich der Nachkriegszeit

Susanne Kogler, Julia Mair, Juliane Oberegger, Johanna Trummer

Erich Marckhl - Musikausbildung in der Steiermark nach 1945.

Brüche und Kontinuitäten

\section{Marie-Anne Kohl}

Die weinende Jury. "Geschlechtslose" Tränen bei globalen Musik-Castingshows?

\section{Fabian Kolb}

Tanztheater und filmische Ästhetik. Cineastische Einflüsse und Gestaltungsweisen in den Kompositionen für die Ballets Suédois 1920-1925

\section{Christian Lehmann}

Tempobezeichnungen von Julius Stockhausen für Die schöne Müllerin:

Ein Quellenfund

\section{Martin Link}

Signum et gens - Zur Gendersemiotik in Clara und Robert Schumanns Liederzyklus Liebesfrühling

\section{Livio Marcaletti}

„Strafspiel" und satirische Stilmittel in musikdramatischen Gattungen des frühen 18. Jahrhunderts

\section{Tobias Marx, Martin Lissner}

Thüringer Musikszene - Jugendmusikredaktionen als außerschulische musikbezogene Bildungskontexte

\section{Maho Naito}

Die Parallelität der Entstehungsprozesse der ersten beiden Symphonien Gustav

Mahlers: Instrumentation, Revision und Dirigierpraxis

\section{Elisa Novara}

Eine Schumann-Werkstatt? Zur Übertragbarkeit der Methoden vom Projekt 
Theodora Oancea, Joachim Pollmann, Jonas Spieker

Kollaborateure - Involvierte - Profiteure. Erarbeitung eines Online-Lexikons zur

Musik in der NS-Zeit

\section{Kiron Patka}

„Ich wollte eigentlich Sängerin werden." Berufsselbstbilder von Tontechniker*innen im Radio

\section{Siegwart Reichwald}

Die Leiden der jungen Clara: Das Klaviertrio Opus 17 als Ausdruck einer Neu-

Romantikerin

\section{Elisa Ringendahl}

Lied versus Oper - Pole musikalischer Gattungen bei Oscar Bie

\section{Benedikt Schubert}

Struktur und Exegese. Über Eigentümlichkeiten in der Arie "Des Vaters Stimme ließ sich hören" (BWV 7/4)

Uwe Seifert, Sebastian Klaßmann, Timo Varelmann, Nils Dahmen

Computational Thinking in der Musikwissenschaft: Jupyter Notebook als Umgebung

für Lehre und Forschung

\section{Yusuke Takamatsu}

Synthese als Modus der Prozessualität bei Schubert:

Sein spezifisches Wiederholungsprinzip im langsamen Satz

\section{Daniel Tiemeyer}

Johann Nepomuk Hummels Sonate in fis-Moll Op. 81 - Studien zu Entstehungs-

hintergrund, Rezeption und formalerStruktur

\section{Andrea van der Smissen}

Musikalische Innovation im Umfeld der Moderne und historischen Avantgarde in Ungarn

\section{Tim Ziemer, Holger Schultheis}

Psychoakustische Sonifikation zur Navigation in bildgeführter Chirurgie

\section{Magdalena Zorn}

Musik mit dem Radio hören: Über den Begriff der musikalischen Aufführung 
Gabriele Buschmeier in memoriam 


\section{Vorwort}

Die vorliegenden Bände dokumentieren die Jahrestagung der Gesellschaft für Musikforschung 2019. In den dreieinhalb Tagen vom 23. bis zum 26. September 2019 wurden in Paderborn und Detmold nicht weniger als 185 Beiträge präsentiert, verteilt auf diverse Symposien, Round tables, Freie Sektionen und Postersessions. Sie alle auf einen Nenner bringen zu wollen, ist ein Ding der Unmöglichkeit - und das ist gut so, ist es doch Ziel der Jahrestagungen, die große Vielfalt der Themen und Methoden des Faches Musikwissenschaft abzubilden. Um die thematische Vielfalt der freien Referate angemessen abbilden zu können und gleichzeitig den inhaltlichen Schwerpunkten der beiden hier publizierten Hauptsymposien ausreichend Raum bieten zu können, erscheinen diese in drei Bänden.

„Musikwissenschaft: Aktuelle Perspektiven": Der Titel der kleinen Reihe ist keine Verlegenheitslösung. Musikwissenschaft im Kontext der Digital Humanities; Musikwissenschaft und Feminismus; Musik und Medien; Musikalische Interpretation - schon die Felder, die von den vier Hauptsymposien bespielt wurden, wären noch vor wenigen Jahrzehnten allenfalls an der Peripherie das Faches zu finden gewesen. Sie entsprechen Arbeitsschwerpunkten der Lehrenden am Musikwissenschaftlichen Seminar der Universität Paderborn und der Hochschule für Musik Detmold, das die Tagung ausrichtete. Zugleich nehmen sie Bezug auf aktuelle Ereignisse und Entwicklungen. So erwuchs das von Andreas Münzmay und Joachim Veit organisierte Symposium „Brückenschläge - Informatik und Musikwissenschaft im Dialog" unmittelbar aus den Erfahrungen im Virtuellen Forschungsverbund Edirom (ViFE) und im fakultäten- und hochschulübergreifenden Zentrum Musik-Edition-Medien (ZenMEM). Der 200. Geburtstag von Clara Wieck/Schumann war der Anlass für das von Rebecca Grotjahn geleitete Symposium „Die Begleiterin - Clara Schumann, Lied und Liedinterpretation", das in enger Kooperation mit der Hochschule für Musik Detmold durchgeführt wurde. Das Hauptsymposium „Brückenschläge" wird in einem separaten Band publiziert (Bd. 3 der vorliegenden Reihe). Im Rahmen dieses Symposiums führte die von Stefanie Acquavella-Rauch geleitete Fachgruppe Digitale Musikwissenschaft eine Posterpräsentation durch, die von den Beiträger*innen erfreulicherweise zu kürzeren Texten umgearbeitet wurden, sodass sie hier ebenfalls, zusammen mit den Postern,

publiziert werden können. Hinzu kommen einige Beiträge, die bereits bei der Jahrestagung 2018 in Osnabrück präsentiert wurden. Auch das Hauptsymposium "Die Begleiterin" wird in einem eigenen Band (Bd. 2) publiziert. Die Beiträge zu den beiden anderen Hauptsymposien hingegen werden an anderen Orten veröffentlicht; in Band 1 („Freie Beiträge zur Jahrestagung der Gesellschaft für Musikforschung 2019") der vorliegenden Publikation finden sich jedoch Einführungen und Abstracts. Das Symposium „Komponieren für das Radio" unter Leitung von Antje Tumat und Camilla Bork (Katholieke Universiteit Leuven) behandelte Einflüsse des Mediums auf Kompositionsprozesse sowie durch radiophone Kompositionen bzw. radiophonen Klang ausgelöste Diskurse. Sarah Schauberger und Cornelia Bartsch (Universität Oldenburg) nahmen das 25-jährige Jubiläum der Fachgruppe Frauen- und Genderstudien zum Anlass für einen Generationenaustausch zum Thema "Musikwissenschaft - Feminismus - Kritik": Was wa- 
ren vor einem Vierteljahrhundert Inhalte und Aufgaben einer feministischen Musikwissenschaft und wie kann sich diese heute positionieren?

Bewusst haben wir im Tagungsbericht auf inhaltliche Eingriffe in die Beiträge verzichtet. ${ }^{1}$ Das gilt besonders für die Freien Referate: Es galt, den Charakter der Jahrestagung als Forum für ,freie', d. h. innovative und auch experimentelle Gedanken zu wahren. Einige Kolleg*innen, die die Tagung mit Vorträgen und Posterpräsentationen bereichert hatten, haben sich gegen eine Publikation im vorliegenden Band entschieden - sei es, weil sie eine Möglichkeit fanden, ihre Beiträge in einem inhaltlich passenderen Rahmen zu veröffentlichen, sei es, weil ihre Überlegungen in ihre entstehenden Qualifikationsschriften fließen sollen, oder sei es, weil sie von den Autor*innen in der vorgetragenen Form zunächst verworfen wurden. Auch damit erfüllt eine Freie-Referate-Sektion ihren Zweck: Die Diskussionen mit der versammelten Fach-Öffentlichkeit sollen dabei helfen, Gedanken weiterzuentwickeln und zu verändern. In diesem Sinne sei allen Beteiligten - den Autor*innen, den nichtpublizierenden Referent*innen und den Mit-Diskutant*innen - ganz herzlich gedankt für ihr Mitwirken bei der Tagung.

Unser herzlicher Dank gilt einer Reihe weiterer Personen, die zum Gelingen dieser drei Bände beigetragen haben. Hier ist besonders Jonas Spieker zu nennen, der uns tatkräftig bei der Redaktion geholfen hat. Andrea Hammes (SLUB Dresden) sei herzlich für die Aufnahme unseres Bandes auf musiconn.publish gedankt - wir freuen uns, damit unsererseits zur Etablierung dieser innovativen Publikationsplattform beizutragen.

Erneut möchten wir an dieser Stelle allen Menschen danken, die uns bei der Organisation, Ausrichtung und Finanzierung der Tagung selbst unterstützt haben: der Präsidentin der Universität Paderborn, Prof. Dr. Birgitt Riegraf, dem Rektor der Hochschule für Musik Detmold, Prof. Dr. Thomas Grosse, den Kolleginnen und Kollegen der beiden beteiligten Hochschulen, dem Vorstand der Gesellschaft für Musikforschung, der Universitätsgesellschaft Paderborn und allen Sponsoren. Besonders dankbar sind wir den Mitarbeiter*innen und den studentischen bzw. wissenschaftlichen Hilfskräften des Musikwissenschaftlichen Seminars, die bei der Vorbereitung und Ausrichtung der Tagung immensen Einsatz zeigten - stellvertretend sei an dieser Stelle Johanna Imm erwähnt, die zusammen mit Nina Jaeschke das Herz des Organisationsteams bildete.

Wir widmen diese Reihe Dr. Gabriele Buschmeier, dem langjährigen Vorstandsmitglied der Gesellschaft für Musikforschung, die kurz vor der Publikation dieses Bandes unerwartet verstarb.

Detmold, im September 2020

Rebecca Grotjahn und Nina Jaeschke

Zitation: Rebecca Grotjahn und Nina Jaeschke, „Vorwort”, in: Freie Beiträge zur Jahrestagung der Gesellschaft für Musikforschung 2019, hrsg. von Nina Jaeschke und Rebecca Grotjahn (= Musikwissenschaft: Aktuelle Perspektiven. Bericht über die Jahrestagung der Gesellschaft für Musikforschung 2019 in Paderborn und Detmold, Bd. 1), Detmold 2020, S. IX-X, DOI: 10.25366/2020.43.

1 Freigestellt war den Autor*innen auch, ob sie sich für eine gendersensible Sprache entscheiden bzw. welche Form des Genderns sie bevorzugen. 